Article

\title{
The Role of the Thoracic Spine during Breathing in Osteogenesis Imperfecta: A Combined Traditional Morphometry and 3D Geometric Morphometrics Research
}

\author{
José María González-Ruiz ${ }^{1, * \mathbb{D}}$, Carlos A. Palancar ${ }^{1}$, Federico Mata Escolano ${ }^{2,3}$, Susanna Llido ${ }^{2}$, \\ Isabel Torres-Sanchez ${ }^{4}$, Francisco García-Río ${ }^{4,5}$, Markus Bastir ${ }^{1,2}$ and Juan A. Sanchis-Gimeno ${ }^{2}$ \\ 1 Virtual Morphology Lab, Museo Nacional de Ciencias Naturales-Consejo Superior de Investigaciones \\ Científicas, J.G. Abascal 2, 28006 Madrid, Spain; palancar.carlos@gmail.com (C.A.P.); \\ mbastir@mncn.csic.es (M.B.) \\ 2 Giaval Research Group, Department of Anatomy and Human Embryology, Faculty of Medicine, \\ University of Valencia, Avda. Blasco Ibanez 15, 46010 Valencia, Spain; fmata@eresa.com (F.M.E.); \\ susanna.llido@uv.es (S.L.); juan.sanchis@uv.es (J.A.S.-G.) \\ 3 ASCIRES ERESA Group, CT and MRI Unit, Avda. de Campanar 114, 46015 Valencia, Spain \\ 4 Hospital Universitario La Paz Institute for Health Research (IdiPAZ), Paseo de la Castellana 261, \\ 28046 Madrid, Spain; maribel.torres.sanchez@gmail.com (I.T.-S.); francisco.garcia@uam.es (F.G.-R.) \\ 5 Centro de Investigación Biomédicaen Red en Enfermedades Respiratorias (CIBERES), Av. de Monforte de \\ Lemos 5, 28029 Madrid, Spain \\ * Correspondence: josemaria.gonzalezr@estudiante.uam.es
}

check for updates

Citation: González-Ruiz, J.M.; Palancar, C.A.; Mata Escolano, F; Llido, S.; Torres-Sanchez, I.;

\section{García-Río, F.; Bastir, M.;}

Sanchis-Gimeno, J.A. The Role of the Thoracic Spine during Breathing in Osteogenesis Imperfecta: A Combined Traditional Morphometry and 3D Geometric Morphometrics Research. Osteology 2022, 2, 1-10. https://doi.org/10.3390/ osteology2010001

Academic Editor: Takahiro Niikura

Received: 15 September 2021

Accepted: 21 December 2021

Published: 23 December 2021

Publisher's Note: MDPI stays neutral with regard to jurisdictional claims in published maps and institutional affiliations.

Copyright: () 2021 by the authors. Licensee MDPI, Basel, Switzerland. This article is an open access article distributed under the terms and conditions of the Creative Commons Attribution (CC BY) license (https:// creativecommons.org/licenses/by/ $4.0 /)$.

\begin{abstract}
OsteogenesisImperfecta (OI) is a rare disease with respiratory problems, which are usually attributed to the secondary effects of scoliosis and rib fractures and to severe restrictive pulmonary disease. Conventional morphometry has already been studied in OI patients but three-dimensional geometric morphometrics (3D GMM) has never been used to assess how the thoracic spine shape changes during maximal breathing. A total of 6 adult subjects with OI type III and 16 healthy controls underwent a spirometric study and two computed tomography scans in maximal inspiration and expiration. Shape data by means of 3D GMM and Cobb angle values of scoliosis and kyphosis were obtained and their relationship with spirometric values was analysed using regressions and mean shape comparisons. No differences in kyphosis $(p=0.285)$ and scoliosis Cobb values $(p=0.407)$ were found between inspiration and expiration in OI patients. The 3D GMM analysis revealed significant shape differences between OI and control subjects $(p<0.001)$ that were related to the inspiration $(p=0.030)$ and not to the expiration $(p=0.079)$. Nevertheless, no significant relation was found between thoracic spine shape, scoliosis, kyphosis and breathing outcomes in both OI patients and controls. There were thoracic spine shape differences during maximal breathing between OI patients and controls that were mainly related to the inspiration.
\end{abstract}

Keywords: geometric morphometrics; kyphosis; scoliosis; osteogenesis imperfecta; thoracic spine shape

\section{Introduction}

Osteogenesis Imperfecta (OI) is a rare disease that affects 1 in every 200,000 individuals [1]. It is characterized by some heterogeneous disorders of connective tissue, especially affecting collagen synthesis [2-4], the presence of bone fractures being common [5]. Sillence et al. [6] classified OI patients in four subtypes (I-IV) but additional types have been described, as knowledge about OI genetics has increased [7]. In this context, OI Type III subjects present short stature and severe and progressive deformations [8]. In addition, bone fragility in OI patients was related to low bone mineral density that it is highly frequent in vertebral bodies $[9,10]$ and could be incremented because of ligament laxity [4], gait deficiencies [11] and delay in motor skills acquisition [9]. 
It is also known that OI patients present vertebral deformities especially in the midthoracic region (TH6, TH7, TH8 and TH9) [12] and the relationship between these deformities and the pulmonary function has been studied [13,14]. According to Watanabe et al. [3], scoliosis is a high-prevalence disorder in these patients, whose motor abilities, breathing skills and self-care capacities are compromised because of its progressive character. In addition, growth has a crucial impact in developing symptoms in Type III patients [9]. At the respiratory functional level, an altered breathing pattern in severe OI is present since childhood and it worsens with age [15]. Life expectancy in Type III is reduced because of cardiopulmonary insufficiency $[13,15]$ but only a few authors have reported data on relations between thorax deformities and pulmonary function [14].

Patients with OI and scoliosis have also associated hyperkyphosis (HK) and this condition is especially prevalent among subjects with OI type III [4,8]. In addition, severe HK has been reported in adulthood in all types of OI [15].

Widmann et al. [16] described a strong correlation of thoracic scoliosis with decreased predicted vital capacity but not in case of thoracic kyphosis. According to LoMauro et al. [13], rib cage deformities have important consequences on ventilation at rest but the role played by thoracic spine remains unclear. Previous kinematic analyses have been carried out during quiet breathing, focusing on scoliosis and ribcage deformity [15]. Sanchis-Gimeno et al. [14] analysed, in OI patients, the consequences of rib cage deformities in pulmonary function but the role of the thoracic spine during maximal inspiration and maximal expiration remained unclear and currently there are no data about the differences in thoracic spine shape between OI patients and healthy subjects during breathing.

As a result, we aimed to test the possible differences in thoracic spine shape between OI Type III patients and healthy subjects during maximal breathing (maximal inspiration and maximal expiration) in a research study that combined conventional morphometry with three-dimensional geometric morphometrics (3D GMM).

\section{Materials and Methods}

\subsection{Subjects}

In total, 6 adult subjects diagnosed with OI Type III confirmed by genetic testing and 16 healthy adult subjects free of any spinal and respiratory pathology from La Paz Hospital (Table 1) were recruited after approval of the research protocol by the local Ethics Committee of our Institution (approval n. H1417174744011). All control subjects were non-smokers. Then, written informed consent was obtained from each participant from both groups. Patients with any other metabolic bone disease except OI and those who underwent surgery for correction of ribcage and/or spinal deformities were excluded. All patients were receiving bisphosphonate therapy.

\subsection{Pulmonary Function Tests and CT Protocol}

Spirometry was performed on both OI Type III and controls by trained clinicians in accordance with the Spanish Society of Pulmonology and Thoracic Surgery guidelines [17] and equations of the Global Lung Function Initiative (https:/ / www.ers-education.org/ guidelines/global-lung-function-initiative/spirometry-tools.aspx, accessed on 14 December 2018) were used as reference values. Individual measures of forced vital capacity (FVC) and forced expiratory volume in $1 \mathrm{~s}$ (FEV1), expressed as absolute value and as percentage of predicted value, are shown in Table 1.

After spirometry was carried out, the participants underwent two CT scans: the first scan in maximal inspiration and the second scan in maximal expiration. Both spirometry and CT scans studies were carried out in order to analyse their clinical evolution and participation was voluntary after being advised about the radiation exposure during CT scans. 
Table 1. Anthropometric and spirometric data of patients with OI and controls. All variables are expressed as median values and interquartile range.

\begin{tabular}{|c|c|c|c|c|c|}
\hline \multicolumn{6}{|c|}{ Description of the Samples Analysed } \\
\hline & \multicolumn{2}{|c|}{ Osteogenesis Imperfecta } & \multicolumn{2}{|c|}{ Control Sample } & \multirow[b]{2}{*}{$p$-value ** } \\
\hline & Median & $\mathrm{IQR} *$ & Median & $\mathrm{IQR} *$ & \\
\hline Age (years) & 42 & $31.5-53.3$ & 58 & $50.3-64.8$ & 0.012 \\
\hline Bodyheight (cm) & 135 & $118.8-145.5$ & 173.5 & $169.5-176.9$ & $<0.001$ \\
\hline Bodyweight (kg) & 49.5 & $40.5-55.8$ & 82 & $72.6-87.4$ & 0.002 \\
\hline $\begin{array}{l}\text { Body mass index } \\
\qquad\left(\mathrm{kg} / \mathrm{m}^{2}\right)\end{array}$ & 29.3 & $22.7-34.0$ & 26.7 & $24.9-28.3$ & 0.396 \\
\hline FVC (L) & 2.1 & $1.2-2.6$ & 4.9 & $3.9-5.1$ & $<0.001$ \\
\hline FEV1 (L) & 2 & $1.1-2.4$ & 3.9 & $2.9-4.1$ & 0.001 \\
\hline FVC, \% pred. (\%) & 89.5 & $70.9-119.7$ & 109.8 & $103.7-118.3$ & 0.150 \\
\hline FEV1, \% pred. (\%) & 98.8 & $77.5-136.0$ & 109.31 & $104.4-112.8$ & 0.580 \\
\hline
\end{tabular}

The 3D mesh segmentation was carried out in 3D Slicer [18]. The algorithms used for spine segmentation from CT scans werebased on binary thresholding filters, with values ranging between 350 and 3024, accurate for bone segmentation [19].

\subsection{Traditional Morphometric Analyses}

We used the Cobb method [20] to assess the kyphotic and scoliotic curvature in our sample. Using Mimics 8 software, we produced reconstructed digital 2D images of the thoracic spines in the mid-sagittal plane from each of the subject's CT scans. We then applied the same technique as from a conventional radiograph to measure the Cobb angle [21]. The direct method (2 lines) was chosen instead of the indirect method (4 lines) to minimize measurement error and the non-strict method (T4-T12 kyphosis) wasselected considering physiological levels of vertebral curvatures instead of the strict method (T1-T12 kyphosis), according to the recommendations of Tanguay et al. [22]. For scoliosis measurement, the inferior endplate of caudal vertebra of thoracic curve and the superior endplate of cranial vertebra were chosen as inferior and superior limits. In order to evaluate the reliability of our measurements, the Cobb angle was measured 3 different times by the same researcher, the intraclass correlation coefficient (ICC) was estimated and their $95 \%$ confident intervals (CI) were calculated based on a 2-way mixed-effect model. ICC values less than 0.5 are considered to be indicative of poor reliability, values between 0.5 and 0.75 indicate moderate reliability, values between 0.75 and 0.9 indicate good reliability and values greater than 0.90 indicate excellent reliability [23].

\subsection{Shape Data and Geometric Morphometrics}

We used 3D GMM [24] to test for statistical differences in thoracic spine shape between OI patients and healthy subjects during maximal inspiration and maximal expiration. Homologous landmarks were previously defined and measured in Viewbox 4.0 (www.dhal.com, accessed on 7 June 2018). In total, 10 landmarks and 10 slide curve semilandmarks [24] were used in each thoracic vertebra along the entire thoracic spine as previously reported [14,25]. Three-dimensionallandmark data of all subjects were subjected to general Procrustes analysis (GPA) [26], a superimposition method that removes all information related to size, orientation and position for statistical comparisons of 3D thoracic spine shape differences between inspiration and expiration and between OI and control groups. 
Regressions and mean shape comparisons were performed in MorphoJ 1.06d [27] and all visualizations were produced using landmark-driven thin-plate spline transformations of 3D surface meshes using the Evan toolkit (http:/ / www.evan-society.org/, 9 December 2018) [28]. Data were entered and stored in an MSExcel file and then transferred to PAST 3.25 [29] for statistical analysis. Continuous variables were presented as median and interquartile range. Differences between groups werecalculatedwith the Mann-Whitney test. Two-sided $p<0.05$ was considered statistically significant.

The measurement error of the landmarks' digitizing process was obtained by measuring one random patient 5 times during 5 non-consecutive days and testing if the Procrustes distance between all measures (0.013) was lesser than the Procrustes distance to 5 randomly selected control patients $(0.014)$, a condition observed in our measures.

\section{Results}

Table 2 presents the kyphosis and scoliosis values obtained in both OI and control subjects. No differences in thoracic kyphosis Cobb values were found between inspiration and expiration in both OI patients $(p=0.285)$ and control subjects $(p=0.597)$. In addition, no differences in scoliosis Cobb values were found between inspiration and expiration $(p=0.407)$ in OI patients.

Table 2. Median Cobb angles and interquartile range of kyphosis and scoliosis measured in the maximal inspiration and maximal expiration moment. Scoliosis was not measured in controls due to its absence among these subjects.

\begin{tabular}{|c|c|c|c|c|}
\hline \multicolumn{5}{|c|}{ Cobb Angle Values Obtained in Subjects Analysed } \\
\hline \multicolumn{5}{|c|}{ Osteogenesis Imperfecta } \\
\hline & \multicolumn{2}{|c|}{ Thoracic Kyphosis } & \multicolumn{2}{|c|}{ Thoracic Scoliosis } \\
\hline & Median * & $\mathrm{IQR} * *$ & Median * & $\mathrm{IQR} * *$ \\
\hline Inspiration & 28.5 & $22.1-31.9$ & 22.5 & $11.3-33.7$ \\
\hline Expiration & 29.8 & $23.9-35.2$ & 15.2 & $11.5-33.1$ \\
\hline$p$-value & \multicolumn{2}{|c|}{0.285} & \multicolumn{2}{|c|}{0.407} \\
\hline \multicolumn{5}{|c|}{ Control Subjects } \\
\hline & \multicolumn{2}{|c|}{ Thoracic Kyphosis } & \multicolumn{2}{|c|}{ Thoracic Scoliosis } \\
\hline & Median * & $\mathrm{IQR} * *$ & Median * & $\mathrm{IQR} * *$ \\
\hline Inspiration & 28.9 & $24.3-33.6$ & - & - \\
\hline Expiration & 30.8 & $24.7-34.5$ & - & - \\
\hline$p$-value & \multicolumn{2}{|c|}{0.597} & \multicolumn{2}{|c|}{-} \\
\hline
\end{tabular}

The ICC used to assess measurement accuracy for the thoracic kyphosis measurements; this was 0.960 , with a CI95\% from 0.919 to 0.982 , in inspiration and 0.913 , with a CI95\% from 0.825 to 0.961 , in expiration, which reflects an excellent reliability in both inspiration and expiration. The ICC, used to assess measurement accuracy for the thoracic scoliosis measurements, was 0.972 , with a CI95\% from 0.920 to 0.991 , and 0.967 , with a CI95\% from 0.918 to 0.991 , in both inspiration and expiration, respectively, which also reflects an excellent reliability. 
The analysis of the correlation between the spirometry values and the kyphosis values revealed no significant correlation between the kyphosis values and the FVC, FEV1, FVC\% pred. and FEV1\% pred. in both OI patients and controls (Table 3).

Table 3. Correlation analysis of spirometric data and Cobb angle of kyphosis in both samples, measured in maximal inspiration and maximal expiration images.

\begin{tabular}{ccccc}
\hline \multicolumn{5}{c}{ Correlation Analysis between Spirometry Values and Kyphosis Values } \\
\hline & \multicolumn{4}{c}{ Inspiration } \\
\hline & Osteogenesis Imperfecta & \multicolumn{2}{c}{ Control Subjects } \\
\hline & $r$ & $p$-value & $r$ & $p$-value \\
\hline FVC (L) & -0.039 & 0.942 & -0.311 & 0.241 \\
\hline FEV1 (L) & -0.016 & 0.976 & -0.171 & 0.527 \\
\hline FVC, \% pred. (\%) & 0.617 & 0.192 & -0.043 & 0.875 \\
\hline FEV1, \% pred. (\%) & 0.570 & 0.238 & 0.197 & 0.465 \\
\hline & & Expiration & \\
\hline & Osteogenesis Imperfecta & Control Subjects \\
\hline FVC (L) & $r$ & $p$-value & $r$ & $p$-value \\
\hline FEV1 (L) & 0.597 & 0.211 & -0.328 & 0.215 \\
\hline FVC, \% pred. (\%) & 0.637 & 0.177 & -0.195 & 0.470 \\
\hline FEV1, \% pred. (\%) & 0.734 & 0.097 & -0.029 & 0.915 \\
\hline
\end{tabular}

FVC, forced vital capacity; FEV1, forced expiratory volume in $1 \mathrm{~s}, \mathrm{FVC} \%$ pred., forced vital capacity expressed as percentage of predicted value; FEV $1 \%$ pred., forced expiratory volume in $1 \mathrm{~s}$ expressed as percentage of predicted value.

Nevertheless, we found significant correlations between the scoliosis values in inspiration and the FVC $(r=0.896 ; p=0.016)$ and FEV1 $(r=0.875 ; p=0.022)$, although not in case of FVC $\%$ pred. ( $r=0.560 ; p=0.248)$ and FEV1\% pred. $(r=0.503 ; p=0.309)$ in OI patients. In addition, we found no significant correlation between the scoliosis values in expiration and the FVC $(r=0.606 ; p=0.202)$, FEV1 $(r=0.605 ; p=0.203)$, FVC $\%$ pred. $(r=0.417 ; p=0.410)$ and FEV1\% pred. $(r=0.363 ; p=0.480)$ in OI patients.

The 3D GMM analysis revealed that the mean difference in theProcrustes distance between inspiration and expiration was 0.035 ( $p=0.977$; Mann-Whitney test) in OI patients and 0.007 ( $p=0.999$; Mann-Whitney test) in control subjects, being significant the difference between OI and control subjects ( $p<0.001$; Mann-Whitney test). This difference in shape between OI patients and controls seemed to be more related to the inspiration ( $p=0.030$; Mann-Whitney test), rather than to the expiration ( $p=0.079$; Mann-Whitney test). The mean shapes of the thoracic spine of patients and controls are shown in maximum inspiration in Figure 1 and in maximum expiration in Figure 2.

In order to explore the quantitative relationship between the thoracic spine shape and the respiratory function, we carried out a regression analysis of our shape data on the respiratory variables in inspiration and expiration (Table 4). None of the regressions were significant. 

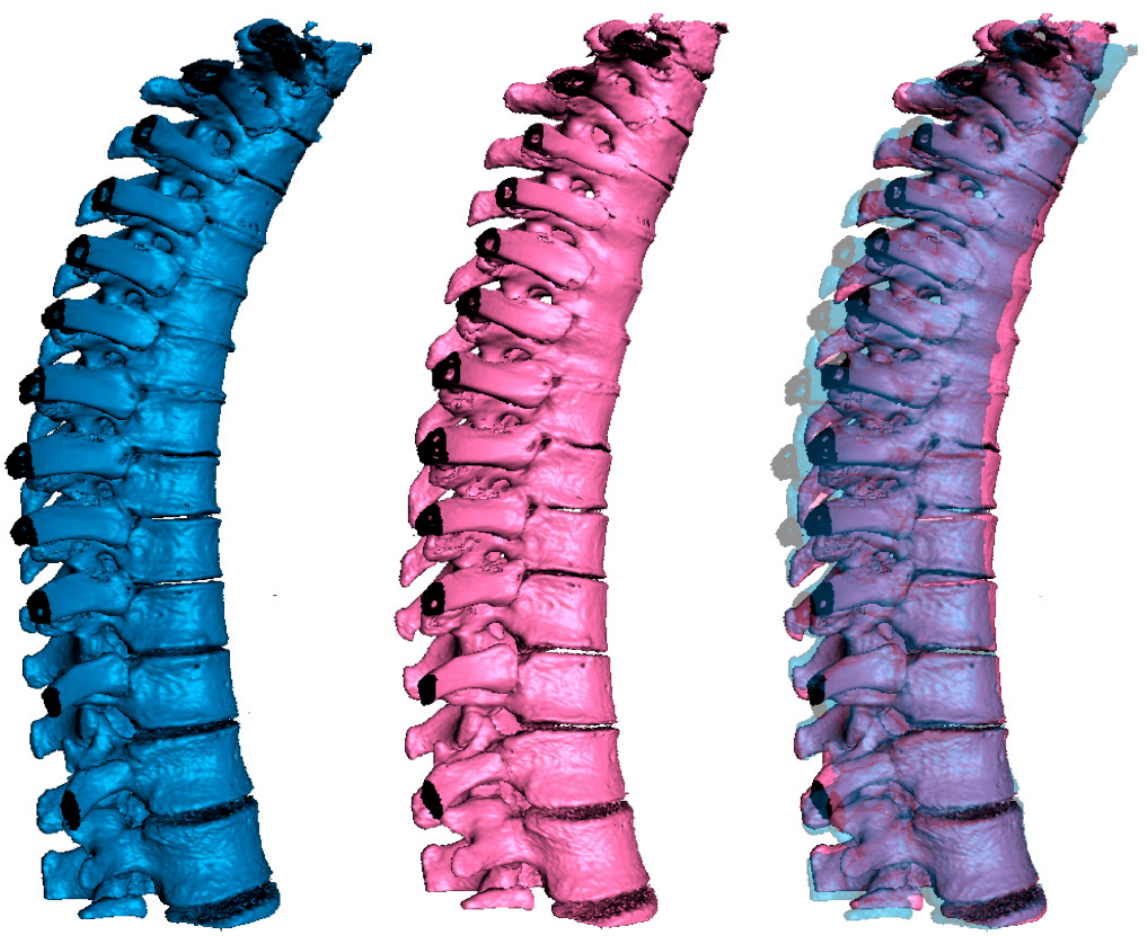

Figure 1. Mean shapes of thoracic spine during maximum inspiration of controls (left, blue) and OsteogenesisImperfecta patients (middle, pink) and the superimposition of both OsteogenesisImperfecta and controls (right, the blue is $50 \%$ transparent in order to show shape differences).
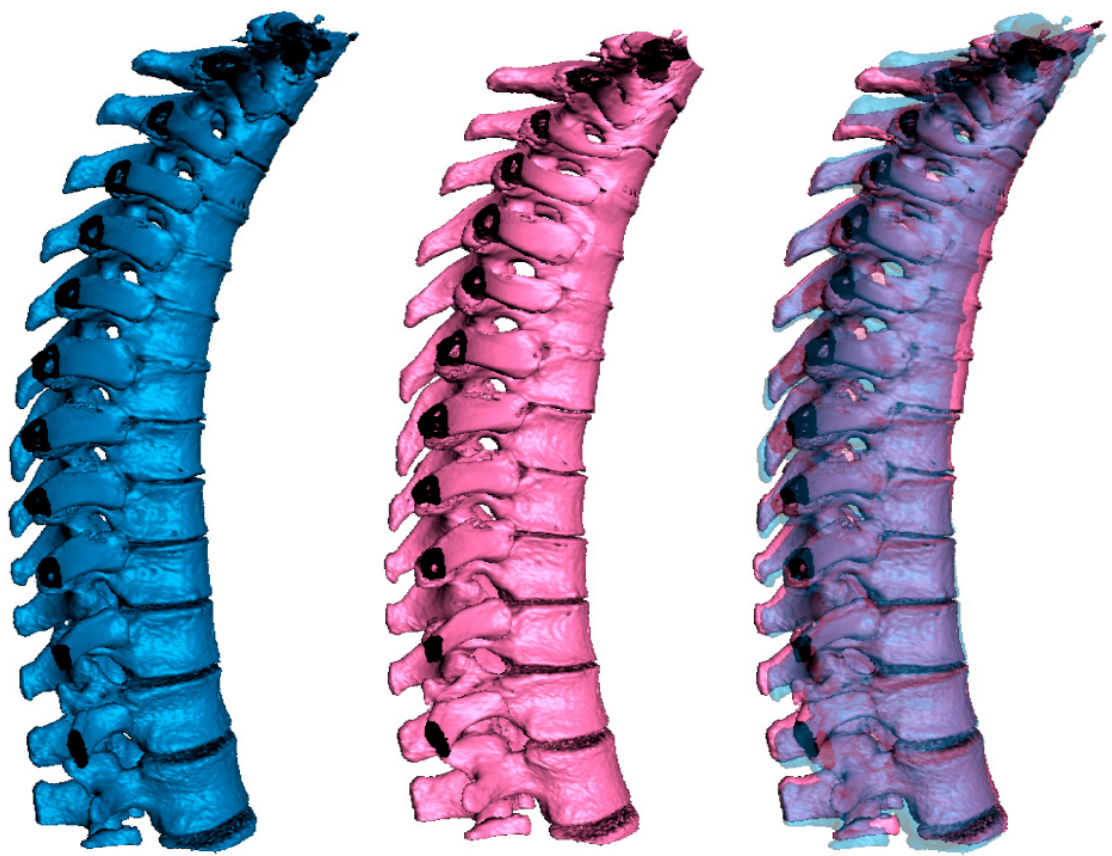

Figure 2. Mean shapes of thoracic spine during maximum expiration of controls (left, blue) and OsteogenesisImperfecta patients (middle, pink) and the superimposition of both OsteogenesisImperfecta and controls (right, the blue is $50 \%$ transparent in order to show shape differences). 
Table 4. Regression analysis of the 3D shape of thoracic spine in maximal inspiration and maximal expiration on spirometric variables.

\begin{tabular}{|c|c|c|c|c|}
\hline \multicolumn{5}{|c|}{ Regression Analysis between Shape Data and Respiratory Variables } \\
\hline \multicolumn{5}{|c|}{ Osteogenesis Imperfecta } \\
\hline & \multicolumn{2}{|c|}{ Inspiration } & \multicolumn{2}{|c|}{ Expiration } \\
\hline & $\%$ predicted & $p$-value & $\%$ predicted & $p$-value \\
\hline FVC (L) & 14.5 & 0.696 & 15.9 & 0.621 \\
\hline FEV1 (L) & 14.8 & 0.674 & 15.6 & 0.651 \\
\hline FVC, \% pred. (\%) & 22.4 & 0.323 & 18.9 & 0.514 \\
\hline FEV1, \% pred. (\%) & 20.5 & 0.376 & 17.6 & 0.588 \\
\hline \multicolumn{5}{|c|}{ Control Subjects } \\
\hline & \multicolumn{2}{|c|}{ Inspiration } & \multicolumn{2}{|c|}{ Expiration } \\
\hline & $\%$ predicted & $p$-value & $\%$ predicted & $p$-value \\
\hline FVC (L) & 4.5 & 0.640 & 5.4 & 0.510 \\
\hline FEV1 (L) & 4.0 & 0.707 & 4.4 & 0.672 \\
\hline FVC, \% pred. (\%) & 3.5 & 0.868 & 3.2 & 0.883 \\
\hline FEV1, \% pred. (\%) & 5.5 & 0.505 & 5.2 & 0.530 \\
\hline
\end{tabular}

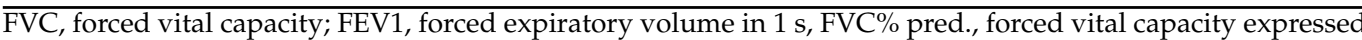
as percentage of predicted value; FEV1\% pred., forced expiratory volume in $1 \mathrm{~s}$ expressed as percentage of predicted value.

\section{Discussion}

Here, we present the first study combining traditional morphometry (Cobb angles of kyphosis and scoliosis) with 3D GMM of the thoracic spine shape changes during maximal inspiration and expiration in OI patients. Previously, the correlation between impaired respiratory function and thoracic spine deformity (scoliosis and spine deformity index) has been investigated $[12,16]$, because respiratory complications are the main cause of death in OI $[30,31]$.

We analysed sixOI Type III patients because the low prevalence of the disorder $[1,8]$ makes the participation of a greater quantity of subjects with OI type III difficult. It seems to be a low number of subjects, but it is in line with the number of patients analysed in previous studies, this fact being a usual limit in studies regarding OI. In this context, in a sample of 73 patients with OI ( 9 type III), Wekre et al. [12] found that vertebral deformities (especially those allocated in the mid-thoracic region) were correlated with FVC and FEV1. The normal ranges for spirometry variables all assume body height to beunperturbed by deformities, which introduces a bias in the evaluation and produces false negative results. To avoid this bias, some authors as Wekre et al. [12] used the arm-span correction devised by Parker et al. [32] to obtain corrected body height as it yields a better evaluation of patients with pulmonary compromise. However, in our study, this was a limit, because the correction by height was only possible in one case as the rest of the patients were unable to extend their arms, which may underlie the absence of significant correlations in our research study.

LoMauro et al. [13] revealed significant reduction in spirometric parameters in type III OI subjects mainly caused by the severity of the disease and sternum deformities (chest wall deformities). It is known that ribcage deformities are more highly correlated than thoracic spine shape with pulmonary function reduction [14] but a study that combines both morphometrical approaches (traditional and 3D) has never been performed. We found significant correlation between scoliosis and FVC/FEV1 values, but not in case of 3D thoracic spine shape, as previously published [14]. However, significant differences were found in thoracic spine shape of OI and controls both in maximal inspiration and expiration in spite of the fact that they are not related to breathing dysfunction. The action of ribcage 
muscles is limited, resulting in poor contribution of pulmonary rib cage to tidal volume [15]. This mechanical disadvantage of the rib cage and the intercostal muscles needs possibly to be compensated at the thoracic spine level in these subjects to achieve maximal inspiration and expiration. Thus, the thoracic spine deforms more during breathing and the diaphragm is required to compensate the reduced function of intercostal muscles [13]. This fact may be related to our findings of greater shape changes in the thoracic spine in OI patients in comparison to controls. Our research study found that OI subjects presented a different deformation of the thoracic spine during maximal breathing, possibly to cover rib cage functional insufficiencies, so we believe that the training of respiratory thoracic muscles could help these patients to improve their breathing skills. In addition, all OI patients were under bisphosphonate therapy, which has been related to the preservation and recovery of the shape of vertebral bodies [33-35]; however, despite this therapy, we found differences in the thoracic spine shape.

Previous analyses were carried out during quiet breathing [13] and only analysed traditional measurements [12] or used 3D GMM [14] but not both traditional and 3D GMM in the same study. As a result, we followed the previous paths and tried to join and combine both methodological approaches (traditional morphometry with 3D GMM). In addition, in our analysis, we considered the maximal breathing performance in inspiration and expiration as FVC and FEV1 test were carried out in maximal physiological requirements. In addition, combining 2D measures (kyphosis and scoliosis) with 3D shapes is required because of the 3D nature of spinal deformities within the human torso [36], but the Cobb angle was measured using CT scan images in supine position because the OI patients were wheelchair users. It is well known that spine curvatures decrease with supine position because of reduction in weight supported by spinal structures [37]. In consequence, Cobb measures could have been underestimated in our study, which is a certain limitation of our results. In addition, this underestimation is expected to be higher in subjects with OI because of hyperlaxity [4] that could have led to over stretched spinal curvatures in lying position.

\section{Conclusions}

In sum, we present, for the first time, that there are thoracic spine shape differences during maximal breathing between OI patients and controls that are mainly related to inspiration rather than expiration.

Author Contributions: J.M.G.-R. (conceptualization, formal analysis, investigation, methodology and writing-original draft), C.A.P. (formal analysis, investigation, methodology, visualization and writing-original draft), F.M.E. (acquisition of data, data analysis/interpretation, critical revision of the article), S.L. (acquisition of data, data analysis/interpretationandcritical revision of the article), I.T.-S. (acquisition of data, data analysis/interpretationandcritical revision of the article), F.G.-R. (acquisition of data, data analysis/interpretationandcritical revision of the article), M.B. (investigation, project administration, supervision, validationandwriting-review and editing), J.A.S.-G. (funding acquisition, project administration, resources, supervision, validation andwriting-review and editing). All authors have read and agreed to the published version of the manuscript.

Funding: This research study was funded by grants of the Ministry of Economy, Industry and Competitiveness (grant number: PID2020-115854GB-I00 (MCINN)), the FundacionEresa (grant number: BF14_005), the Care4Brittlebones Foundation (grant number: OTR2016-15543INVES) and the University of Valencia (grant number: UV-INV_AE18-773873).

Institutional Review Board Statement: The study was conducted according to the guidelines of the Declaration of Helsinki and approved by the Institutional Review Board (or Ethics Committee) of University of Valencia (protocol code H1417174744011).

Informed Consent Statement: Informed consent was obtained from all subjects involved in the study. Written informed consent was obtained from the patient(s) to publish this paper.

Data Availability Statement: Not applicable. 
Conflicts of Interest: The authors declare no conflict of interest. The funders had no role in the design of the study; in the collection, analyses, or interpretation of data; in the writing of the manuscript, or in the decision to publish the results.

\section{References}

1. Marini, J.C.; Blissett, A.R. New genes in bone development: What's new in OsteogenesisImperfecta. J. Clin. Endocrinol. Metab. 2013, 98, 3095-3103. [CrossRef]

2. Byers, P.H.; Steiner, R.D. Osteogenesis Imperfecta. Annu. Rev. Med. 1992, 43, 269-282. [CrossRef]

3. Watanabe, G.; Kawaguchi, S.; Matsuyama, T.; Yamashita, T. Correlation of scoliotic curvature with Z-score bone mineral density and body mass index in patients with Osteogenesis Imperfecta. Spine 2007, 32, 488-494. [CrossRef]

4. Lubicky, J.P. The spine in Osteogenesis Imperfecta. Spine Deform. 2012, 1, 124-132. [CrossRef]

5. Marini, J.C.; Forlino, A.; Bächinger, H.P.; Bishop, N.J.; Byers, P.H.; De Paepe, A.; Fassier, F.; Fratzl-Zelman, N.; Kozloff, K.M.; Krakow, D.; et al. Osteogenesis Imperfecta. Nat. Rev. Dis. Primers 2017, 18, 17052. [CrossRef] [PubMed]

6. Sillence, D.O.; Senn, A.; Danks, D.M. Genetic heterogeneity in Osteogenesis Imperfecta. J. Med. Genet. 1979, 16, 101-116. [CrossRef]

7. Wallace, M.J.; Kruse, R.W.; Shah, S.A. The spine in patients with Osteogenesis Imperfecta. J. Am. Acad. Orthop. Surg. 2017, 25, 100-109. [CrossRef] [PubMed]

8. Martin, E.; Shapiro, J.R. Osteogenesis imperfect: Epidemiology and pathophysiology. Curr. Osteoporos. Rep. 2007, 5, 91-97. [CrossRef]

9. $\quad$ Engelbert, R.H.H.; Uiterwaal, C.S.P.M.; Van der Hulst, A.; Witjes, B.; Helders, P.J.M.; Pruijs, H.E.H. Scoliosis in children with Osteogenesis Imperfecta: Influence of severity of disease and age of reaching motor milestones. Eur. Spine J. 2003, 12, 130-134. [CrossRef]

10. Lee, D.Y.; Cho, T.J.; Choi, I.H.; Chung, C.Y.; Yoo, W.J.; Kim, J.H.; Park, Y.K. Clinical and radiological manifestations of Osteogenesis Imperfecta type V. J. Korean Med. Sci. 2006, 21, 709-714. [CrossRef] [PubMed]

11. Schlösser, T.P.C.; Van der Heijden, G.J.M.G.; Versteeg, A.L.; Castelein, R.M. How "idiopathic" is adolescent idiopathic scoliosis? A systematic review on associated abnormalities. PLOS ONE 2014, 9, e97461.

12. Wekre, L.L.; Kjensli, A.; Aasand, K.; Falch, J.A.; Eriksen, E.F. Spinal deformities and lung function in adults with Osteogenesis Imperfecta. Clin. Respir. J. 2014, 8, 437-443. [CrossRef]

13. LoMauro, A.; Pochintesta, S.; Romei, M.; D'Angelo, M.G.; Pedotti, A.; Turconi, A.C.; Aliverti, A. Rib cage deformities alter respiratory muscle action and chest wall function in patients with severe Osteogenesis Imperfecta. PLoS ONE 2012, 7, e35965. [CrossRef]

14. Sanchis-Gimeno, J.A.; Lois-Zlolniski, S.; González-Ruiz, J.M.; Palancar, C.A.; Torres-Tamayo, N.; García-Martínez, D.; Aparicio, L.; Perez-Bermejo, M.; Blanco-Perez, E.; Mata-Escolano, F.; et al. Association between ribs shape and pulmonary function in patients with Osteogenesis Imperfecta. J. Adv. Res. 2019, 22, 177-185.

15. LoMauro, A.; Fraschini, P.; Pochintesta, S.; Romei, M.; D’Angelo, M.G.; Aliverti, A. Ribcage deformity and the altered breathing pattern in children with Osteogenesis Imperfecta. Pediatr. Pulmonol. 2018, 53, 964-972. [CrossRef] [PubMed]

16. Widmann, R.F.; Bitan, F.D.; LaPlaza, F.J; Burke, S.W.; DiMaio, M.F.; Schneider, R. Spinal deformity, pulmonary compromise, and quality of life in Osteogenesis Imperfecta. Spine 1999, 24, 1673-1678. [CrossRef] [PubMed]

17. Garcia-Rıo, F.; Calle, M.; Burgos, F.; Casan, P.; Del Campo, F.; Galdiz, J.B.; Giner, J.; González-Mangado, N.; Ortega, F.; Puente Maestu, L. Spirometry. Spanish Society of Pulmonology and Thoracic Surgery (SEPAR). Arch. Bronconeumol. 2013, 49, 388-401.

18. Pieper, S.; Halle, M.; Kikinis, R. 3D Slicer. In Proceedings of the 2004 2nd IEEE International Symposium on Biomedical Imaging: Nano to Macro (IEEE Cat No. 04EX821), Arlington, VA, USA, 18 April 2004; pp. 632-635.

19. Bastir, M.; Sanz-Prieto, D.; Burgos, M. Three-dimensional form and function of the nasal cavity and nasopharynx in humans and chimpanzees. Anat. Rec. 2021. [CrossRef]

20. Cobb, J. Outline for the study of scoliosis. Am. Acad. Orthop. Surg. Instr. Course Lect. 1948, 5, 261-275.

21. Brink, R.C.; Wijdicks, S.P.J.; Tromp, I.N.; Schlösser, T.P.C.; Kruyt, M.C.; Beek, F.J.A.; Castelein, R.M. A reliability and validity study for different coronal angles using ultrasound imaging in adolescent idiopathic scoliosis. Spine J. 2017, 18, 979-985. [CrossRef]

22. Tanguay, F.; Mac-Thiong, J.-M.; De Guise, J.A.; Labelle, H. Relation between the sagittal pelvic and lumbar spine geometries following surgical correction of adolescent idiopathic scoliosis. Eur. Spine J. 2006, 16, 531-536. [CrossRef] [PubMed]

23. Koo, T.K.; Li, M.Y. A guideline of selecting and reporting intraclass correlation coefficients for reliability research. J. Chiropr. Med. 2016, 15, 155-163. [CrossRef]

24. Mitteroecker, P.; Gunz, P. Advances in Geometric Morphometrics. Evol. Biol. 2009, 36, 235-247. [CrossRef]

25. González-Ruiz, J.M. La Morfometría Geométrica como Herramienta Aplicada al Estudio de la Escoliosis en Pacientes con Osteogénesis Imperfect. Master's Dissertation, Universidad Autónoma de Madrid, Madrid, Spain, 2018.

26. Rohlf, F.J.; Slice, D. Extensions of the Procrustes Method for the Optimal Superimposition of Landmarks. Syst. Zool. 1990, 39, 40-59. [CrossRef]

27. Klingenberg, C.P. MorphoJ: An integrated software package for geometric morphometrics. Mol. Ecol. Resour. 2011, 11, 353-357. [CrossRef] 
28. Bastir, M.; García-Martínez, D.; Torres-Tamayo, N.; Palancar, C.A.; Fernández-Pérez, F.J.; Riesco-López, A.; Osborne-Márquez, P.; Ávila, M.; López-Gallo, P. Workflows in a Virtual Morphology Lab: 3D scanning, measuring, and printing. J. Anthropol. Sci. 2019, 97, 107-134.

29. Hammer, Ø. PAST Paleontological Statistics; Version 3.15; Natural History Museum, University of Oslo: Oslo, Norway, 2017.

30. McAllion, S.J.; Paterson, C.R. Causes of death in Osteogenesis Imperfecta. J. Clin. Pathol. 1996, 49, 627-630. [CrossRef]

31. Arcaro, G.; Braccioni, F.; Gallan, F.; Marchi, M.R.; Vianello, A. Noninvasive positive pressure ventilation in the management of acute respiratory failure due to Osteogenesis Imperfecta. J. Clin. Anesth. Feb. 2012, 24, 55-57. [CrossRef]

32. Parker, J.M.; Dillard, T.A.; Phillips, Y.Y. Arm span-height relationships in patients referred for spirometry. Am. J. Resp. Crit. Care Med. 1996, 154, 533-536. [CrossRef]

33. Cristofaro, R.L.; Hoek, K.J.; Bonnett, C.A.; Brown, J.C. Operative treatment of spine deformity in Osteogenesis Imperfecta. Clin. Orthop. 1979, 139, 40-48. [CrossRef]

34. Semler, O.; Beccard, R.; Palmisano, D.; Demant, A.; Fricke, O.; Schoenau, E.; Koerber, F. Reshaping of vertebrae during treatment with neridronate or pamidronate in children with Osteogenesis Imperfecta. Horm. Res. Paediatr. 2011, 76, 321-327. [CrossRef] [PubMed]

35. Palomo, T.; Fassier, F.; Ouellet, J.; Sato, A.; Montpetit, K.; Glorieux, F.H.; Rauch, F. Intravenous bisphosphonate therapy of young children with Osteogenesis Imperfecta: Skeletal findings during follow up throughout the growing years. J. Bone Miner. Res. 2015, 30, 2150-2157. [CrossRef] [PubMed]

36. Donzelli, S.; Poma, S.; Balzarini, L.; Borboni, A.; Respizzi, S.; Villafane, J.H.; Zaina, F.; Negrini, S. State of the art of current 3-D scoliosis classifications: A systematic review from a clinical perspective. J. Neuroeng. Rehabil. 2015, 16, 91. [CrossRef] [PubMed]

37. Wessberg, M.D.; Danielson, M.D.; Willén, M.D. Comparison of Cobb angles in idiopathic scoliosis on standing radiographs and supine axially loaded MRI. Spine 2006, 31, 3039-3044. [CrossRef] 